$\mathrm{p}=0.01$ ) when compared to patients with both abnormal SGUS findings and LGS focal lymphocytic sialadenitis.

Conclusion: SGUS and LSG histopathology showed only a moderate correlation in patients with pSS. SGUS may be useful to identify pSS patients with a more severe inflammatory LSG infiltrates, ultimately contributing to promote non-invasive phenotyping of multiple subsets in pSS. Disclosure of Interests: Chiara Baldini: None declared, Francesco Ferro: None declared, Nicoletta Luciano: None declared, Gianmaria Governato: None declared, Marta Mosca Paid instructor for: GlaxoSmithKline, Lilly, UCB, Stefano Bombardieri: None declared, Valentina Donati: None declared

DOI: 10.1136/annrheumdis-2019-eular.6251

\section{THU0260 \\ SYSTEMIC LUPUS ERYTHEMATOSUS AND CYTOPENIAS: THE KEY FINDINGS IN BONE MARROW}

Ana Barrera-Vargas ${ }^{1}$, Jonathan Campos-Guzmán ${ }^{1}$, Samuel Govea-Peláez ${ }^{1}$, Aldo García-Ramos ${ }^{2}$, Roberta Demichelis-Gómez ${ }^{2}$, Christianne Bourlon ${ }^{2}$, Javier Merayo-Chalico ${ }^{1}$, Jorge Alcocer-Varela ${ }^{1} .{ }^{1}$ Instituto Nacional de Ciencias Médicas y Nutrición Salvador Zubirán, Immunology and Rheumatology, Mexico City, Mexico; ${ }^{2}$ Instituto Nacional de Ciencias Médicas y Nutrición Salvador Zubirán, Hematology and Oncology, Mexico City, Mexico

Background: Cytopenias are common in systemic lupus erythematosus (SLE), and it is fundamental to determine their etiology in order to establish an adequate therapeutic strategy.

Objectives: To describe the findings in the bone marrow aspirations (BMA) and biopsies of patients with SLE and cytopenias, as well as clinical and laboratory features associated with the etiology of the hematological abnormalities.

Methods: We performed a retrospective study in a third-level hospital in Mexico City. We included patients who fulfilled ACR criteria for SLE, presented with cytopenias and had a BMA and biopsy performed between 2000 and 2016. We described the main aspirate and biopsy findings, and also analyzed the final diagnosis and its association with clinical, laboratory and serological features.

Results: We included 101 patients; median age was 32 years and $81.2 \%$ were women. Leukopenia $(<3000$ cells $/ \mu l)$ was found in $47.5 \%$ of patients, with $29.7 \%$ having moderate or severe neutropenia $(<1000$ cells/ $\mu \mathrm{l})$. Lymphopenia $(<1000$ cells $/ \mu \mathrm{l})$ was a common finding $(71.3 \%$ of patients). Moderate-to-severe thrombocytopenia $(<50 \mathrm{~K} / \mu \mathrm{l})$ was present in $28.7 \%$ of patients. Finally, $25.8 \%$ of patients presented with pancytopenia. Regarding bone marrow findings, there was erythroid dysplasia in $50.5 \%$ of patients, granulocytic dysplasia in $28.7 \%$ and megakaryocytic dysplasia in $16 \%$. Myelofibrosis was found in $2.6 \%$. An increase in plasma cells $(\geq 5 \%)$ was found in $21.8 \%$.

In $72.3 \%$ of patients, bone marrow interpretation was conclusive. The most common diagnoses were disease activity (24.8\%) and drug-associated myelotoxicity $(28.7 \%)$. When compared to other etiologies, in patients with cytopenias secondary to disease activity, it was more frequent for the bone marrow be hypercellular (56 vs $23 \%, p=0.006$ ) and to have increased megakaryocytes (40 vs $17.4 \%, p=0.048$ ). Conversely, granulocytic dysplasia was less common in this group of patients $(17.4 \%$ vs $54.3 \%, p=0.036$ )

We analyzed factors associated with both activity and toxicity as final diagnoses (Table 1). After multivariate analysis, a neutrophil count $<1000$ cells $/ \mu \mathrm{l}$ was a protective factor for disease activity (OR $0.021 ; 95 \% \mathrm{Cl}$ $0.001-0.428, p 0.012$ ). On the other hand, a history of renal activity (OR 4.3; $95 \% \mathrm{Cl} 1.3-14.2, \mathrm{p}=0.024)$ and neutrophils less than $1000 \mathrm{cells} / \mu \mathrm{l}$ (OR 4.05; $95 \% \mathrm{Cl} 1.15-14.19, \mathrm{p}=0.029$ ) were found to be independent risk factors for myelotoxicity.

Table 1.

\begin{tabular}{|c|c|c|c|}
\hline Characteristic & $\begin{array}{l}\text { Disease } \\
\text { activity } \\
(\mathrm{N}=25)\end{array}$ & $\begin{array}{c}\text { Drug-associated } \\
\text { toxicity } \\
(\mathrm{N}=28)\end{array}$ & $\mathrm{p}$ \\
\hline History of disease activity & $80 \%$ & $86 \%$ & 0.719 \\
\hline - Mucocutaneous & $100 \%$ & $96.4 \%$ & 1 \\
\hline - Hematologic & $44 \%$ & $78.6 \%$ & 0.012 \\
\hline \multicolumn{4}{|l|}{ - Renal } \\
\hline Neutrophils (cells/ $\mu \mathrm{l}$ ) & $16 \%$ & $17.9 \%$ & 0.004 \\
\hline - Mild neutropenia (1000-1499) & $4 \%$ & $7.1 \%$ & \\
\hline & $4 \%$ & $39.3 \%$ & \\
\hline \multicolumn{2}{|l|}{$\begin{array}{l}999) \\
\text { - Severe neutropenia }(<500)\end{array}$} & & \\
\hline Lymphopenia $(<1000$ cells $/ \mu l)$ & $68 \%$ & $70 \%$ & 1 \\
\hline
\end{tabular}

Platelets $(\mathrm{K} / \mu \mathrm{l})$

- Mlld thrombocytopenia (50-99)

- Severe thrombocytopenia $(<50)$

SLEDAI score

SLICC damage index

Treatment

Azathioprine

Mofetil mycophenolate

Hydroxychloroquine

Prednisone

$0 \leq 7.5 \mathrm{mg}$

o $7.6-30 \mathrm{mg}$

$0>30 \mathrm{mg}$

Conclusion: The main causes of cytopenias in our SLE patients were disease activity and bone marrow toxicity. Our findings suggest that when patients present with less than 1000 neutrophils/ul, it is unlikely for the cytopenias to be secondary to disease activity. Our study reinforces the diagnostic utility of BMA and biopsy, and the associations we described may assist clinicians to determine the etiology of cytopenias in SLE patients, in order to make appropriate therapeutic decisions.

Disclosure of Interests: Ana Barrera-Vargas: None declared, Jonathan Campos-Guzmán: None declared, Samuel Govea-Peláez: None declared Aldo García-Ramos: None declared, Roberta Demichelis-Gómez: None declared, Christianne Bourlon: None declared, Javier Merayo-Chalico Speakers bureau: Pfizer, Jorge Alcocer-Varela: None declared DOI: 10.1136/annrheumdis-2019-eular.6515

\section{THU0261 THE URINARY CELLULAR PROFILE AS A BIOMARKER FOR LUPUS NEPHRITIS}

Amira El-Girby ${ }^{1}$, Abeer Abdelati ${ }^{1}$, Hanaa Donia ${ }^{2}$, Nouran Eshak ${ }^{1} .{ }^{1}$ Faculty Of Medicine Alexandria University, Internal Medicine - Rheumatology and Clinical Immunology, Alexandria, Egypt, ${ }^{2}$ Faculty Of Medicine Alexandria University, Clinical Pathology, Alexandria, Egypt

Background: Proliferative lupus nephritis is one of the most common and serious manifestation of SLE and is a major cause of morbidity. A search for the ideal biomarker for $L N$ is still underway, one that can be used for early detection, and correlate with the class \& activity of LN. Urine is normally devoid of leucocytes, however it has been observed that macrophages and T-lymphocytes are routinely present in the urine of LN patients and those with other proliferative renal diseases. ${ }^{(1)}$ This provided the idea for their potential use as biomarkers for proliferative LN. Objectives: To study the urinary CD4+, CD8+ T Lymphocytes, and CD14 + monocytes in patients with proliferative lupus nephritis, and explore their use as a biomarker for $\mathrm{LN}$.

Methods: Our subjects included 30 patients with biopsy proven proliferative LN and 30 SLE patients without clinical or lab evidence of LN as controls. Lab investigations included serum creatinine, urine analysis, protein creatinine ratio, anti-ds DNA Ab, C3 and C4. For the flowcytometric analysis $100 \mathrm{ml}$ of freshly voided urine in a sterile container were obtained from patients and controls. All samples were processed within 2-4 hours of collection to ensure viability of the cells. Urine mononuclear cell count was done using a hemocytometer. The urine samples were centrifuged then washed twice by phosphate-buffered saline/bovine serum albumin (PBS/BSA) and resuspended in about $300 \mu \mathrm{ls}$ PBS. The cells were stained with anti CD8- FITC, anti CD4- PE, anti CD14-PERCP and anti- CD3-APC monoclonal antibodies. The flow cytometric analysis was done using Becton Dickinson, FACS Calibur multi-parameter flow cytometer equipped with BD CellQuest Pro software for data analysis.

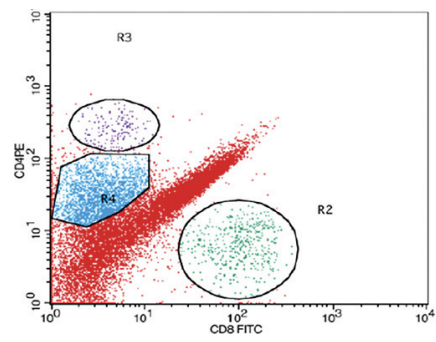

Abstract THU0261 - Figure 1 